\title{
Findings About Events or Interventions Data Collection Day
}

National Cancer Institute

\section{Source}

National Cancer Institute. Findings About Events or Interventions Data Collection Day.

NCl Thesaurus. Code C113361.

The day of the week the findings about an event or intervention was collected. 